\title{
Abstract \\ Integrated Architectural Design: The Role of Software in Collaborating with Engineers
}

\author{
Thomas Seebohm, School of Architecture, University of Waterloo
}

With the increasing importance of designing for sustainability, that is, to leave the environment as least as good or better than before a design intervention, designing holistically is essential. By holistic design is meant a process where all issues ranging from the physical, such as energy consumption and lighting, to social issues, such as how spaces are used, are addressed, not just metaphorically, but in a rigorous and rational manner based on analysis.

The traditional approach would have the architect call on engineering specialists at some stage in the design resulting in a number of problems that prevent holistic design. Among them are the following: 1) the difficulty of revising a design to meet technical requirements once it is too far advanced, 2) the difficulty of communication between architects and engineers regarding design intent, 3 ) the complexity of contemporary projects having outgrown the capability of a single person, the architect, to coordinate alone, and 4) professional bias on the part of the engineering consultants whereby the consultants propose solutions without providing the architect with the full range of alternatives.

A solution to these problems is Integrated Design understood as a collaborative design process whereby engineering and other specialist consultants collaborate with the architect from the very beginning of a project in a mutually supportive, creative and sharing environment. One of the best examples of a firm of specialist consultants practicing in this way is the firm Transolar in Germany, headed my Mathias Schuler. This firm specializes in climate engineering. In collaborations with Transolar all design participants, whether architects, engineers or physicists are considered equal partners in the design process.

While Transolar's collaborations are a success story in terms of the collaborative process and the wonderfully creative buildings that have resulted from this process, it is proposed that to make Integrated Design more effective in general, that architects become conversant in the many discipline-specific software packages that are increasingly available and user-friendly. Ability to use such software packages with their embodied discipline-specific knowledge will allow architects to explore technical possibilities on their own without consultant bias to better prepare the architect for consultant meetings and to be in a better position to talk the same language as the engineering consultants. The paper will review some of the specialist software currently available that the author has used and how this software can assist in the Integrated Design process. As well, implications for the education of the architect will be brought into focus. 